OPEN ACCESS

Edited by:

Jingmin Gu,

Jilin University, China

Reviewed by:

Demeng Tan,

Fudan University, China

Shuai Le

Army Medical University, China

Can Zhang,

Qingdao Agricultural University, China

*Correspondence:

Biao Kan

kanbiao@icdc.cn

Specialty section:

This article was submitted to

Clinical Microbiology,

a section of the journal

Frontiers in Cellular and Infection

Microbiology

Received: 01 February 2021

Accepted: 29 March 2021

Published: 16 April 2021

Citation:

Sun H, Liu M, Fan F, Li Z, Fan Y,

Zhang J, Huang Y, Li Z, Li J, Xu J

and Kan B (2021) The Type II

Secretory System Mediates

Phage Infection in Vibrio cholerae.

Front. Cell. Infect. Microbiol. 11:662344.

doi: 10.3389/fcimb.2021.662344

\section{The Type II Secretory System Mediates Phage Infection in Vibrio cholerae}

\author{
Huihui Sun ${ }^{1,2}$, Ming Liu ${ }^{1}$, Fenxia Fan ${ }^{1}$, Zhe $L_{i}{ }^{1}$, Yufeng Fan ${ }^{1}$, Jingyun Zhang ${ }^{1}$, \\ Yuanming Huang ${ }^{1}$, Zhenpeng $L i^{1}$, Jie $L i^{1}$, Jialiang $X u^{3}$ and Biao Kan ${ }^{1 *}$ \\ 1 State Key Laboratory for Infectious Disease Prevention and Control, National Institute for Communicable Disease Control \\ and Prevention, Chinese Center for Disease Control and Prevention, Beijing, China, ${ }^{2}$ National Institute of Environment Health, \\ Chinese Center for Disease Control and Prevention, Beijing, China, ${ }^{3}$ School of Light Industry, Beijing Technology and \\ Business University, Beijing, China
}

Attachment and specific binding to the receptor on the host cell surface is the first step in the process of bacteriophage infection. The lytic phage VP2 is used in phage subtyping of the Vibrio cholerae biotype El Tor of the $\mathrm{O} 1$ serogroup; however, its infection mechanism is poorly understood. In this study, we aimed to identify its receptor on V. cholerae. The outer membrane protein EpsD in the type II secretory system (T2SS) was found to be related to VP2-specific adsorption to $V$. cholerae, and the T2SS inner membrane protein EpsM had a role in successful VP2 infection, although it was not related to adsorption of VP2. The tail fiber protein gp20 of VP2 directly interacts with EpsD. Therefore, we found that in $V$. cholerae, in addition to the roles of the T2SS as the transport apparatus of cholera toxin secretion and filamentous phage release, the T2SS is also used as the receptor for phage infection and probably as the channel for phage DNA injection. Our study expands the understanding of the roles of the T2SS in bacteria.

Keywords: bacteriophage, type II secretory system, receptor, EpsD, Vibrio cholerae

\section{INTRODUCTION}

Cholera is a severe intestinal infectious disease caused by toxigenic Vibrio cholerae, which still seriously attacks human health in undeveloped countries. Cholera outbreaks and endemics occur mainly due to the ingestion of contaminated water and food (Sack et al., 2004; Weil and Ryan, 2018). Successfully colonized $V$. cholerae in the host small intestine may express a number of virulence factors in response to host signals, including cholera toxin (Reidl and Klose, 2002; Yang et al., 2013; Liu et al., 2016), thus causing vomiting and watery diarrhea symptoms. V. cholerae can be divided into more than 200 serogroups according to the diversity of surface $\mathrm{O}$ antigens; currently, only toxigenic $\mathrm{O} 1$ and $\mathrm{O} 139$ serogroup strains are believed to cause cholera epidemics or pandemics (Faruque et al., 1998), and O1 strains can be further classified into classical biotype and El Tor biotype.

Bacteriophage or phage is a general term for viruses that can infect bacteria, causing lysis or lysogenic conversion of the infected bacterial host according to the characteristics of virulent or lytic phages and temperate phages (Hatfull and Hendrix, 2011; Salmond and Fineran, 2015). Interactions between phages and bacteria result in the modulation and biodiversity of bacterial communities in 
the environment and host. In $V$. cholerae, phages may cause the proliferation and decline of the different clones in the environment and may contribute to the disappearance of epidemics or the appearance of new epidemics caused by new clones (Faruque et al., 2005; Jensen et al., 2006). Lytic phages can also be used in the subtyping of bacteria and even to eliminate drug-resistant pathogens in the clinic (Kazmierczak et al., 2014).

The first step for phages to infect the host bacteria is attachment to the specific receptors of the host cells. Tailed phages use a broad range of receptor-binding proteins to specifically interact with their cognate bacterial cell surface receptors. These receptors are diverse in different host cells, and most components are localized on the bacterial cell surface, such as many porins of the outer membrane, lipopolysaccharide (LPS) (Rakhuba et al., 2010; Bertozzi Silva et al., 2016), and flagella or pili, which can also act as phage receptors (Harvey et al., 2018; McCutcheon et al., 2018). Identifying the receptors of phages may facilitate understanding of the infection and host range of the phages and resistance mutations of the bacteria since a sensitive host may evolve resistance to phages by receptor mutation, for example, under infection pressure from phages.

In a phage typing scheme for the $\mathrm{El}$ Tor biotype of $\mathrm{O} 1$ serogroup $V$. cholerae, five $V$. cholerae lytic phages (named VP1 to VP5 in turn) are used for the subtyping of O1 El Tor strains (Zhang et al., 2009). Lipopolysaccharide and outer membrane proteins have been found to be receptors of some typing phages (Zhang et al., 2009). In this study, we aimed to identify the receptor of the phage VP2. We adopted the transposon library strategy to identify the genes related to VP2 infection in $V$. cholerae and found a type II secretion system (T2SS), which has been identified for its importance as an extracellular protein transport apparatus for the secretion of cholera enterotoxin (CT) and release of the filamentous phage CTXФ (Davis et al., 2000), also playing a vital role for VP2 adsorption and infection processes. Our study revealed a new role of T2SS, which is a common cross-membrane apparatus in many bacteria, and present a new insight for the function of this transporter.

\section{MATERIALS AND METHODS}

\section{Phage, Bacterial Strains, Plasmids, and Media}

VP2 was isolated from the river water of Wenzhou, Zhejiang Province, in 1962. The bacterial strains and plasmids used in this study are listed in Table 1. V. cholerae O1 El Tor 16017 is the host of VP2 and is used for VP2 propagation. The V. cholerae El Tor strain N16961 is sensitive to the phage VP2 and was used as the wild-type strain in this study. For in vitro growth experiments, the bacteria were cultured in LB medium unless otherwise noted. Antibiotics were used at the following concentrations: ampicillin (Amp), $100 \mu \mathrm{g} / \mathrm{mL}$; streptomycin (Sm), $100 \mu \mathrm{g} / \mathrm{mL}$; kanamycin (Kan), $50 \mu \mathrm{g} / \mathrm{mL}$; gentamicin (Gm), $20 \mu \mathrm{g} / \mathrm{mL}$; tetracycline (Tc), $2 \mu \mathrm{g} / \mathrm{mL}$ and chloramphenicol $(\mathrm{Cm}), 30 \mu \mathrm{g} / \mathrm{mL}$ for Escherichia coli but $2 \mu \mathrm{g} / \mathrm{mL}$ for $V$. cholerae.

\section{Screening of VP2-Resistant Mutants}

E. coli SM10גpir (Chiang and Mekalanos, 2000) bearing the plasmid pSC123 (Simon et al., 1983) named SM10-123, was used as the donor strain, and conjugation was performed by using the

TABLE 1 | Strains and plasmids used in this study.

\begin{tabular}{|c|c|c|}
\hline Strains and plasmids & Relevant properties & Reference \\
\hline \multicolumn{2}{|l|}{ V. cholerae } & Laboratory \\
\hline$\overline{N 16961}$ & Spontaneous mutant of N16961, Inaba, Sm ${ }^{r}$ & collections \\
\hline $\mathrm{N}-\Delta$ epsM & epsM (VC2724) deletion of N16961, Smr & This study \\
\hline $\mathrm{N}-\Delta \mathrm{eps} D$ & epsD (VC2733) deletion of N16961, $\mathrm{Sm}^{r}$ & This study \\
\hline $\mathrm{N}-\Delta e p s \mathrm{M}-\mathrm{C}$ & $\mathrm{N}-\Delta$ epsM strain complemented with pSRKTc-EpsM & This study \\
\hline $\mathrm{N}-\Delta e p s D-\mathrm{C}$ & $\mathrm{N}-\Delta e p s D$ strain complemented with pSRKGm-EpsD & This study \\
\hline \multicolumn{3}{|l|}{ E. coli } \\
\hline 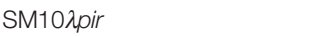 & Kan, thi thr leu tonA lacY supE recA::RP4-2-TC::Muגpir & \\
\hline DH5 $\alpha \lambda$ pir & 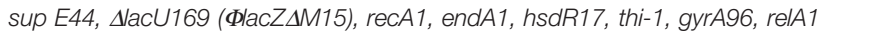 & \\
\hline XL1blue & endA1 gyrA96 thi-1 hsdR17 supE44 relA1 lac & \\
\hline BTH101 & F-, cya-99, araD139, galE15, galK16, rpsL1 (Strr), hsdR2, mcrA1, mcrB1 & \\
\hline \multicolumn{3}{|c|}{ (2) - } \\
\hline pSC123 & Suicide plasmid carrying transposon; $\mathrm{Kan}^{r} \mathrm{Cm}^{r}$ & \\
\hline pWM91 & Suicide plasmid; oriR oriT lacZ tetAR sacB & \\
\hline pWM91- & pWM91 carrying upstream and downstream fragments flanking epsM & This study \\
\hline pWM91- $\Delta$ eps $D$ & pWM91 carrying upstream and downstream fragments flanking epsD & This study \\
\hline pUT18C (abbreviated as pT18C) & pUT18C-derived vector, designed to create C-terminal heterologous protein fusion, $\mathrm{Amp}^{r}$ & \\
\hline pKT25 (abbreviated as pT25) & lac promoter and the T25 fragment for C-terminal heterologous protein fusion. Kan $^{r}$ & \\
\hline pSRKTc & lac promoter and $\mid a c l^{q}$, Tet $^{r}$ & \\
\hline pSRKTc-EpsM & pSRKTc-derived, VC2724 (epsM), Tet $^{r}$ & This study \\
\hline pSRKGm-EpsD & pSRKGm-derived, VC2733 (epsD), Tet $^{r}$ & This study \\
\hline pT25-EpsD & pKT25-derived, epsD, Kan ${ }^{r}$ & This study \\
\hline pT18C-gp20 & pUT18C-derived, gp20, Amp ${ }^{r}$ & This study \\
\hline pGEX-4T-1-EpsD & pGEX-4T-1-derived, epsD, Amp ${ }^{r}$ & This study \\
\hline pET30a-gp20 & pET30a(+)-derived, gp20, Kan $^{r}$ & This study \\
\hline
\end{tabular}


VP2-sensitive $V$. cholerae strain N16961 as the recipient to obtain a transposon insertion library. The transposon mutation pool was mixed with a VP2 suspension $\left(10^{8} \mathrm{PFU} / \mathrm{mL}\right)$ at a ratio of $1: 5$ and incubated for $10 \mathrm{~min}$ at $37^{\circ} \mathrm{C}$. The resulting cultures were spread onto LB agar plates with Kan and Sm. The resistant strains were subsequently verified by double-layer plaque assay as described previously (Frost et al., 1999), and the confirmed nonlysed strains were selected as candidates for verifying transposon insertion location.

Arbitrary PCR (Judson and Mekalanos, 2000) was performed with two rounds to identify the transposon insertion site on the chromosome. In the first round, the chromosomal DNA of candidates was used as amplification templates, and the primers ARB-1, ARB-6, and 123-3 (Table 2) were used in amplification. PCR was performed under the following conditions: $95^{\circ} \mathrm{C}$ for $5 \mathrm{~min}$; six cycles of $94^{\circ} \mathrm{C}$ for $30 \mathrm{~s}, 30^{\circ} \mathrm{C}$ for $30 \mathrm{~s}$, and $72^{\circ} \mathrm{C}$ for $1 \mathrm{~min}$; 30 cycles of $94^{\circ} \mathrm{C}$ for $30 \mathrm{~s}, 55^{\circ} \mathrm{C}$ for $30 \mathrm{~s}$, and $72^{\circ} \mathrm{C}$ for $1 \mathrm{~min}$; and $72^{\circ} \mathrm{C}$ for $5 \mathrm{~min}$. The second round of PCR amplified the PCR product of the first round with the primers 123-4 and ARB-2 (Table 2). Amplification conditions were as follows: 30 cycles of $94^{\circ} \mathrm{C}$ for $30 \mathrm{~s}, 55^{\circ} \mathrm{C}$ for $30 \mathrm{~s}$, and $72^{\circ} \mathrm{C}$ for $1 \mathrm{~min}$, followed by $72^{\circ} \mathrm{C}$ for $5 \mathrm{~min}$. The amplicons were sequenced using the primer 123-4. The transposon insertion sites were confirmed by comparing the sequencing results with the N16961 reference sequence.

\section{Construction of Gene Deletions and Complementation}

In-frame deletions were constructed by cloning the regions flanking target genes into the suicide vector pWM91 containing a $s a c B$ counterselectable marker (Metcalf et al., 1996). The

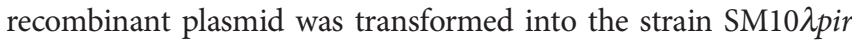
and introduced into $V$. cholerae N16961 by conjugation. Doublecrossover recombination mutants were selected using $10 \%$ sucrose plates at $22^{\circ} \mathrm{C}$ and confirmed via PCR and sequencing. The plasmids overexpressing eps $M$ and epsD were obtained by cloning the complete eps $M$ into a pSRKTc plasmid and epsD genes into a pSRKGm plasmid (Khan et al., 2008).

The primers used for the construction are listed in Table 2.

\section{VP2 Lysis Assay and Efficiency-of-Plating Assay}

A double-agar overlay plaque assay (Korotkov and Sandkvist, 2019) was used to examine the lysis status of VP2 on V. cholerae. Briefly, $100 \mu \mathrm{L}$ of exponential cultures of wild-type and eps $M$ and eps $D$ deletion mutant strains and their complements were mixed with $0.6 \%$ soft agar medium and poured on top of bottom agar. Ten microliters from a phage suspension containing approximately $10^{8} \mathrm{PFU}$ was spotted in the middle of the lawn of bacteria, incubated overnight at $37^{\circ} \mathrm{C}$ and imaged. Each strain experiment was repeated three times. Bacterial strains were considered sensitive to the phage if the degree of lysis was observed as complete clearing. In contrast, bacterial strains were considered resistant if there was no effect of the phage on bacterial growth. When indicated, $500 \mu \mathrm{M}$ IPTG was included in the medium to induce gene expression.

The efficiency-of-plating (EOP) assay was also conducted to quantitate the lysis ability of VP2 as previously described with some modifications (Yang et al., 2020). In brief, the wild-type strain, eps $M$ and epsD deletion mutant strains and their

TABLE 2 | Primers used in this study.

\begin{tabular}{|c|c|c|}
\hline Primer & Sequence $\left(5^{\prime}-3^{\prime}\right)^{a}$ & Destination \\
\hline VC2724-UP-Xhol-5' & CCGCTCGAGAGTGGTACCGGTAGCGGTAG & pWM91-sepsM \\
\hline VC2724-UP-3' & CAAGTAAGGAGAACAGCCGCGCGGCGAAATGAT & \\
\hline VC2724-DOWN-5' & CGCCGCGCGGCTGTTCTCCTTACTTGGGCTTCA & \\
\hline VC2724-DOWN-Notl-3' & AAGGAAAAAAGCGGCCGCCCAAGAGTGGCGTTATGAGC & \\
\hline VC2733-UP-Xhol-5' & CCGCTCGAGTGGCCATCACTITGACCCGT & \\
\hline VC2733-UP-3' & GTTCCCAGTGAAACAACCAGCTCGCAAGTGGAA & \\
\hline VC2733-DOWN-5' & TGCGAGCTGGTTGTTCACTGGGAACTCCCTTG & pWM91- $-\Delta e p s D$ \\
\hline VC2733-DOWN-Notl-3' & AAGGAAAAAAGCGGCCGCCGACTTGGAGCCCCACGTTA & \\
\hline $123-3$ & TCACCAACTGGTCCACCTAC & arbitrary PCR \\
\hline $123-4$ & CGCTCTTGAAGGGAACTATG & \\
\hline ARB1 & GGCCACGCGTCGACTAGTACNNNNNNNNNNGATAT & \\
\hline ARB6 & GGCCACGCGTCGACTAGTACNNNNNNNNNNACGCC & \\
\hline ARB2 & GGCCACGCGTCGACTAGTAC & \\
\hline EpsM- Ndel -F & GGAATTCCATATGATGAAAGAATTATTGGCTCCTGTG & pSRKTc-EpsM \\
\hline EpsM- Xbal -R & GCTCTAGATCAGCCTCCACGCTTCAGTTG & \\
\hline EpsD-Xbal-F & GCTCTAGACATTGCTTGGCTTCCATCTG & \\
\hline EpsD-Ndel-R & GGAATTCCATATGAGGGAGTTCCCAGTGAAATA & EpsD \\
\hline gp20-BamHI-F & CGCGGATCCCATGACTATCCAGAACAAAGAACC & pT18-gp20 \\
\hline gp20-Kpnl-R & CGGGGTACCCGAACGTCCGTAAAATACAAGTT & \\
\hline nT25-EpsD-BamHI-F & CGCGGATCCCAACGAGTITAGCGCCAGCTाT & pT25-EpsD \\
\hline nT25-EpsD-Kpnl-R & CGGGGTACCCGTTGCTTGGCTTCCATCTGCTCAA & \\
\hline EpsD-BamHI-F & CGCGGATCCCGCCGTGCGCAAGTGTTGA & pGEX-4T-1-EpsD \\
\hline EpsD-Xhol-R & CCGCTCGAGTCATTGCTTGGCTTCCATCTGC & \\
\hline gp20-BamHI-F & CGCGGATCCATGACTATCCAGAACAAAGAA & pET-30a-gp20 \\
\hline gp20-HindllII-R & CCCAAGCTITAAACGTCCGTAAAATACAAG & \\
\hline
\end{tabular}

${ }^{a}$ Restriction sites are underlined. 
complements $\left(10^{8} \mathrm{CFU} / \mathrm{mL}\right)$ were mixed with $10^{6} \mathrm{PFU} / \mathrm{mL}$ concentrated VP2, incubated for $15 \mathrm{~min}, 10$-fold dilutions of the mixtures were prepared with LB. For plating, $100 \mu \mathrm{l}$ of diluents of the mixtures was add to $4 \mathrm{ml}$ of $0.6 \%$ top agar and poured on the bottom agar, and the plate was incubated at $37^{\circ} \mathrm{C}$ overnight. The EOP value was calculated as the ratio of the number of lysis plaques produced on the bacterial lawn of the target to the number of plaques produced on the lawn of the host strain.

\section{Phage Adsorption Assays}

A titer of at least $10^{6} \mathrm{PFU} / \mathrm{mL}$ concentrated VP2 was mixed separately with different $V$. cholerae variants $\left(10^{8} \mathrm{CFU} / \mathrm{mL}\right)$, incubated for $3 \mathrm{~min}, 5 \mathrm{~min}$ and $10 \mathrm{~min}$ at $37^{\circ} \mathrm{C}$, and then centrifuged at $6,000 \mathrm{rpm}$ for $8 \mathrm{~min}$. The residual phage titers of the supernatant were counted in $10^{-3}$ dilutions and tested by double-layer plaque assay. Phage without N16961 treatment was used as a control.

\section{Cloning, Expression and Purification of the VP2 Tail Filament Protein and T2SS EpsD}

For a bacterial two-hybrid assay, the recombinant plasmids pKT25-epsD and pUT18C-gp20 were constructed as previously described (Fan et al., 2018). Briefly, two putative interacting proteins (EpsD and gp20) were genetically fused to two complementary fragments, T25 and T18, of the active domain of adenylate cyclase (CyaA) from Bordetella pertussis (Ladant, 1988). First, the epsD truncated sequence (lacking the signal peptide, encoding 25-674 aa) and gp20 complete sequence were amplified using the N16961 and VP2 genomes as templates, respectively. The PCR products of eps $D_{25-674}$ and gp20 were digested with the BamHI and KpnI restriction enzymes and ligated into the corresponding pKT25 and pUT18C vectors digested with the same enzymes. The resulting plasmids were cotransformed into the E. coli $\Delta c y a$ mutant BTH101 for subsequent study. All primers used are included in Table 2.

For pull-down assays, the truncated EpsD and complete gp20 genes were subcloned into prokaryotic expression vectors to obtain the corresponding proteins. A gene fragment of epsD (encoding 340-675 aa) was cloned into the pGEX-4T-1 vector and introduced into the overexpression strain E. coli BL21 (DE3), and protein purification was performed as described previously (Howard et al., 2019). Full-length gp20 was inserted into the pET-30a vector and induced in E. coli strain BL21 (DE3). Cells containing the pET-30a-gp20 recombinant plasmid were grown in $\mathrm{LB}$ medium until the $\mathrm{OD}_{600}$ reached $0.5-0.6$, and $0.5 \mathrm{mM}$ IPTG was added to induce expression overnight at $16^{\circ} \mathrm{C}$. Cells were harvested, and the pellets were resuspended in buffer A (20 $\mathrm{mM}$ Tris- $\mathrm{HCl}, \mathrm{pH} 9.0,300 \mathrm{mM} \mathrm{NaCl}$, supplemented with protease inhibitors). The cells were lysed by sonication and centrifuged at $12,000 \mathrm{rpm}$ for $30 \mathrm{~min}$ at $4^{\circ} \mathrm{C}$. The His6-tagged protein gp20 was purified using $\mathrm{Ni}^{2+}$ resin (Invitrogen), and the elution samples were dialyzed in buffer B $(20 \mathrm{mM}$ Tris- $\mathrm{HCl}, \mathrm{pH}$ 9.0, $300 \mathrm{mM} \mathrm{NaCl}$ ) overnight and were used in interaction assays with GST-tagged EpsD. The protein concentration was determined using Pierce's BCA Protein Assay Reagent Kit. All primers and restriction enzymes used are listed in Table 2.

\section{Binding Capacity of vp2 Tail Filament Protein With Wild-Type N16961 and N- $\Delta$ epsD}

We fused gp20 to a His6 tag, mixed it with an Alexa Fluor 488conjugated anti-His-tag monoclonal antibody, incubated it in the dark at room temperature and resuspended it in filter-sterilized phosphate-buffered saline (PBS). After the unbound antibodies were washed out fully with PBS, they were mixed with wild-type strains and the mutant strain $\mathrm{N}-\Delta e p s D$. Antibodies and no antibodies were added to these two strains as controls. Samples were then analyzed using BD flow cytometry.

\section{Bacterial Two-Hybrid System for Analysis of the EpsD and gp20 Interaction}

The cyaA mutant E. coli BTH101 strain containing pKT25-epsD and pUT18C-gp20 was cultured overnight and then subcultured to $\log$ phase in $\mathrm{LB}$ medium with shaking at $220 \mathrm{rpm}$ at $37^{\circ} \mathrm{C}$. The $\beta$ galactosidase activities were measured in the presence of different concentrations of isopropyl-beta-D-thiogalactopyranoside (IPTG) as the inducer as previously described (Yang et al., 2013). The leucine zipper of GCN4 (Karimova et al., 1998) was used as a positive control.

\section{$\mathrm{Ni}^{2+}$-Affinity Pull-Down of gp20 and EpsD}

Mixtures of $0.3 \mathrm{mg}$ of His6-tagged gp20 protein and $0.1 \mathrm{mg}$ of GST-tagged EpsD protein were rotated at room temperature for $2 \mathrm{~h}$, bound to $\mathrm{Ni}^{2+}$ resin and incubated for $1 \mathrm{~h}$ at $4^{\circ} \mathrm{C}$. Mixtures of His6-tagged gp20 protein and GST protein were used as negative controls.

$\mathrm{The}^{2} \mathrm{Ni}^{2+}$ resin was washed extensively to remove nonspecifically bound proteins, and then the bound proteins were eluted with elution buffer containing $300 \mathrm{mM}$ imidazole, $10 \mathrm{mM}$ Tris- $\mathrm{HCl}$ and $500 \mathrm{mM} \mathrm{NaCl}$ ( $\mathrm{pH}$ 8.0). Five micrograms of each sample were submitted to $12 \%$ SDS-PAGE and Western blot analysis. Unless otherwise noted, all samples were boiled for $10 \mathrm{~min}$ in SDS loading buffer before separation. After electrophoresis, proteins on the gels were transferred to PVDF membranes (Immobilon-P, Millipore). Both mouse anti-GST and anti-His6 tag monoclonal antibodies (Tiangen Biotech, Beijing) were used in the Western blot analysis. An anti-mouse peroxidase-conjugated AffiniPure IgG $(\mathrm{H}+\mathrm{L})$ secondary antibody (Zhong Shan Jin Qiao, Beijing) was used for protein detection.

\section{RESULTS AND DISCUSSION}

\section{Components of the Type II Secretion System Were Involved in VP2 Infection}

To identify $V$. cholerae genes related to the resistance to VP2 infection phenotype, a selection strategy of phage-resistant mutants generated by transposon insertion was used. The pool of transposon mutants from the VP2-sensitive strain N16961 was generated by conjugation with SM10-123 carrying the plasmid pSC123. Then, the phage VP2 
was added into this pool to lyse the mutant cells sensitive to VP2 infection, whereas the mutants resistant to VP2 survived. Possible resistant colonies were selected on agar and further confirmed for resistance to VP2 infection with double-layer agar. Eighteen candidates resistant to VP2 were collected. Each candidate was amplified through arbitrary PCR, and the amplicon was sequenced to verify the transposon location on the chromosome of the resistant strains. A total of six different insertion sites were identified, including in the coding sequences of the genes VCA0904 ( H+/gluconate symporter and related permease), VC1936 (phosphatidate cytidylyltransferase), VC0718 (DNA recombination-dependent growth factor C), VC0420 (conserved hypothetical protein CHP02099), VCA0863 (lipase, Pla-1/cef, extracellular), and VC2724 (EpsM). Among these genes, VC2724 is located in the gene cluster of the T2SS, correspondingly coding for the inner membrane protein EpsM, which is located in the membrane (Figure 1A). For the phage receptor identification purpose of this study, we first selected the membrane-located protein EpsM as a candidate.

We further constructed the mutant strain $\mathrm{N}-\Delta e p s M$ with inframe deletion of eps $M$ from the wild-type strain N16961 and the complement strain $\mathrm{N}-\Delta e p s M-\mathrm{C}$ carrying the intact eps $M$ gene in the pSRKTc plasmid (Table 2). Phage lysis assays with both strains showed that $\mathrm{N}-\Delta e p s M$ was not lysed by VP2 (Figure 1B), whereas the strain $\mathrm{N}-\Delta e p s M-\mathrm{C}$ carrying complementary eps $M$ restored the sensitivity to VP2 (Figure 1B). EOP assay was consistent with the phage lysis assays (Figure 1B). Both assays showed that the $V$. cholerae T2SS protein EpsM is needed for VP2 infection.

\section{The Outer Membrane-Localized Protein EpsD of T2SS Could Adsorb VP2}

T2SS is a cell envelope-spanning macromolecular complex that is prevalent in gram-negative bacterial species and serves as the predominant virulence mechanism of many bacteria (Johnson et al., 2006). The system is composed of a core set of highly conserved proteins that assemble an inner membrane platform, a periplasmic pseudopilus and an outer membrane complex termed the secretin (Johnson et al., 2006). The model of the T2SS machine includes an outer membrane protein, the secretin $\mathrm{EpsD}$, the first subdomain of which is related to domains in phage tail filament proteins and outer membrane TonB-dependent receptors (Johnson et al., 2006). We suspected that EpsD might be involved in the interaction of $V$. cholerae with the phage VP2. Subsequently, we constructed the epsD deletion strain $\mathrm{N}-\Delta e p s D$ and the complementary strain $\mathrm{N}-\Delta e p s D-\mathrm{C}$ (containing intact eps $\mathrm{D}$ in the plasmid pSRK-Gm) to determine their sensitivities to phage VP2. VP2 infection assays showed that the epsD mutant strain could not be lysed by VP2, while the strain N- $\Delta e p s D-C$ could be lysed (Figure 1B), showing the important role of EpsD in VP2 infection; therefore, it could be expected that EpsD possibly acts as the receptor for VP2.

The role of EpsD in VP2 infection was also determined by phage adsorption assays to detect the binding ability of the phage VP2 to the epsD and eps $M$ mutant strains and their corresponding complementary strains. VP2 particles were mixed with the wildtype strain N16961 for $3 \mathrm{~min}$, and after centrifugation, the remaining supernatant had a much lower phage titer than the control, which was determined by a PFU assay (Figure 2), showing that the sensitive strain N16961 had strong adsorption of VP2.

A

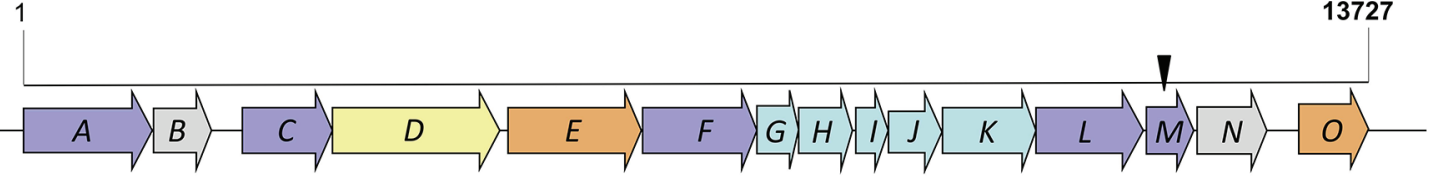

eps gene cluster

B

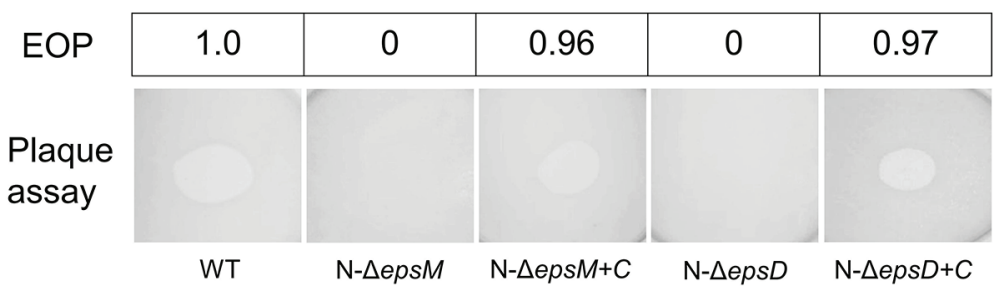

FIGURE 1 | Transposon insertion and VP2 infection of the mutants. (A) Transposon insertion site of VP2-resistant mutants in the gene cluster of the T2SS in N16961. The eps genes are colored according to the classification of functions and positions of the proteins in T2SS in V. cholerae (Korotkov et al., 2012), including inner-membrane plateform proteins (light blue), outer-membrane secretin (yellow), pseudopilin (light green), others (light brown) and unknown (grey). The black arrow represents the site where the transposon was inserted into the epsM. (B) Detection of VP2 infection in V. cholerae mutants by double-layer plaque assay and EOP assay. The wild-type V. cholerae El Tor strain N16961 was used as the control for plaque formation. N- $\Delta$ epsM showed VP2 resistance (no plaque formation). The strain $\Delta$ epsM-C, carrying the EpsM expression plasmid cloned into the strain N- $\Delta$ epsM, was sensitive to VP2. N- $\Delta$ epsD showed VP2 resistance (no plaque formation). The strain $\Delta e p s D-C$, carrying the EpsD expression plasmid cloned into the strain $\mathrm{N}-\Delta e p s D$, was sensitive to VP2. The values of EOP were shown in the top half of the figure. 


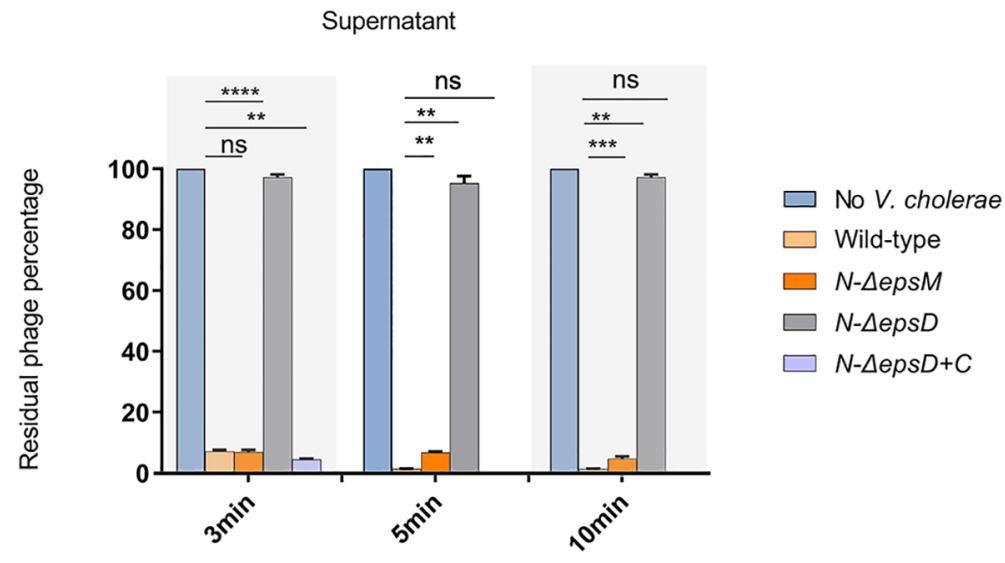

FIGURE 2 | VP2 adsorption by the wild-type strain N16961 and its mutants. The VP2 phage (10 $\left.{ }^{6} \mathrm{CFU} / \mathrm{mL}\right)$ was mixed with fresh N16961 culture, N- $\Delta$ epsM, $\mathrm{N}-\Delta e p s D, \mathrm{~N}-\Delta e p s D+\mathrm{C}\left(10^{8} \mathrm{CFU} / \mathrm{mL}\right)$ respectively for $3 \mathrm{~min}, 5 \mathrm{~min}, 10 \mathrm{~min}$ at $37^{\circ} \mathrm{C}$, and then, each sample was centrifuged at $6000 \mathrm{rpm}$ for $8 \mathrm{~min}$. LB culture medium containing only VP2 phage was used as a negative control, and the phage titer in the control supernatant was set to $100 \%$. The experiment was repeated three times. The mean of three independent assays was shown and error bars represent the standard deviation. ${ }^{\star \star} P=0.0026,{ }^{\star \star \star} P=0.0001,{ }^{\star \star \star \star} P<0.0001$, ns, no significance (Student's $t$ test).

When VP2 was mixed with the strain $\mathrm{N}-\Delta e p s D$, its obvious VP2 titer difference in the supernatant was found when compared to the sensitive strain N16961 (Figure 2), indicating that VP2 did not bind to the epsD mutant. Such adsorption could be restored by complementing the plasmid carrying intact epsD into $\mathrm{N}-\Delta e p s D$ (strain N- $\Delta e p s D-C$, Figure 2). The VP2 adsorption efficiency of the eps $M$ deletion strain $\mathrm{N}-\Delta e p s M$ was similar to that of the wild-type strain N16961. These data suggested that EpsD could adsorb VP2 but EpsM could not, which could be explained by their different membrane locations in the T2SS apparatus in V. cholerae.

\section{VP2 Adsorbed to EpsD of V. cholerae Through Its Tail Fiber Protein gp20}

The genome of VP2 has been sequenced previously in our laboratory (GenBank accession number: NC_005879). Here, we submitted the VP2 genome sequence to RAST (http://rast.nmpdr. org/rast.cgi) for the prediction of open reading frames (ORFs). The ORF $g p 20$ was predicted as the phage tail fiber protein gene, and then we expressed this protein as the ligand to detect its interaction with $V$. cholerae. To observe the binding ability of gp20 to the wildtype strain N16961 and N-AepsD, the expressed His6-tagged gp20 was labeled with an anti-His6 fluorescent antibody, mixed with the wild-type strain $\mathrm{N} 16961$ and mutant strain $\mathrm{N}-\Delta e p s D$, and then analyzed using flow cytometry to measure the geometric mean fluorescence intensity (MFI). The MFI in the tests of the His6tagged gp20/N16961 mixture was much higher than that of the His6-tagged gp20/N- $\Delta e p s D$, and the MFI of the latter was similar to that of the controls, representing the background fluorescence intensity, as shown in Figure 3, which indicated that no gp20 adsorbed to the epsD deletion mutant.

\section{The VP2 Tail Protein gp20 Interacted Directly With EpsD}

To determine the possible interaction between $V$. cholerae EpsD and gp20 of VP2, we performed mutual interaction assays between these two proteins by using a bacterial two-hybrid (BACTH) approach (Karimova et al., 1998). In this study, the recombinant plasmids pKT25-epsD and pUT18C-gp20 were constructed and co-transformed into the E. coli $\Delta c y a$ mutant BTH101. The results showed that $0.5 \mathrm{mM}$ IPTG could induce the maximum $\beta$-galactosidase activity, similar to that of $1.0 \mathrm{mM}$ IPTG induction and the positive control (Figure 4A), suggesting that EpsD could interact with gp20.

In addition, pull-down assays were carried out to detect the interaction between gp20 and EpsD. His-tagged gp20 was immobilized on $\mathrm{Ni} 2+$ resin to capture GST-tagged EpsD. In the elution buffer, GST-tagged EpsD could be detected by Western blot (Figure 4B), further showing that gp20 can bind directly to EpsD.

Overall, we demonstrated that in $V$. cholerae, the outer membrane protein EpsD of T2SS acts as the receptor in phage VP2 infection. As an important secretion apparatus, T2SSs are widespread among gram-negative bacteria and contribute to pathogenesis in the host and environmental survival (Cianciotto and White, 2017; Korotkov and Sandkvist, 2019). The T2SS transports chitinase, lipase, hemagglutinin/protease and other proteases in some bacteria (Sandkvist, 2001; Evans et al., 2008; Sikora et al., 2011). V. cholerae may secrete cholera toxin (Sikora et al., 2011) and even mediate the release of the filamentous phage CTXФ through EpsD (Davis et al., 2000). In E. coli, the membrane protein PulD of the T2SS contributes to phage extrusion across the membrane and assembly of phages (Genin and Boucher, 1994; Russel, 1998; Sandkvist, 2001). In our study, in addition to these roles of T2SS, we showed that it may mediate lytic phage infection by the specific adsorption of VP2 to the outer membrane component EpsD. EpsM is a part of the interface between the regulating part and the rest Eps proteins of the T2SS (Abendroth et al., 2004). Combined with the dot assays and the phage adsorption assays, the inner membrane protein 


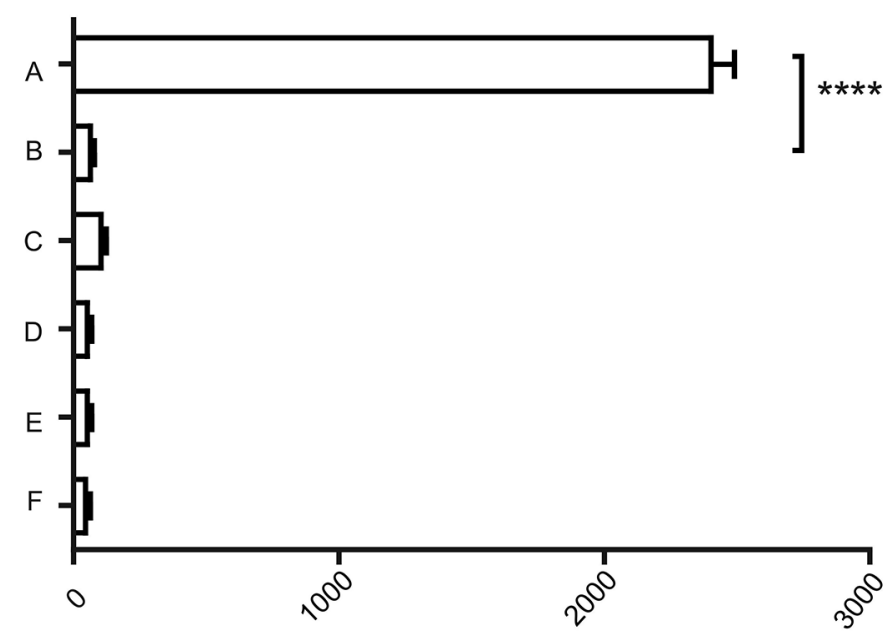

MFI

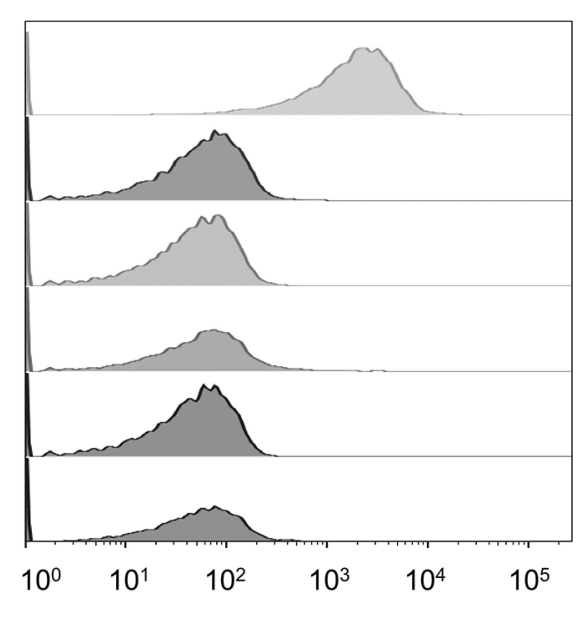

FITC-A

FIGURE 3 | Binding capacity of the vp2 tail filament protein with wild-type N16961 and N- $\Delta$ epsD. (A): Wild type+ His6-tagged gp20+anti-His6 tag antibody; (B): N- $\Delta$ epsD + His6-tagged gp20+anti-His6 tag antibody; (C): wild type +anti-His6 tag antibody; (D): N- $\Delta e p s D$ +anti-His6 tag antibody; (E): wild type; (F): N- $\Delta$ epsD. Ten micrograms of His6tagged gp20 protein and $10 \mu \mathrm{g}$ of Alexa Fluor 488-conjugated anti-His-tag monoclonal antibody (product \# MA1-21315-A488) were mixed and incubated for 30 min in the dark at room temperature and then transferred to the wild-type $\mathrm{N} 16961$ and $\mathrm{N}$ - $\Delta$ eps $D$ strains, with $\mathrm{OD} 600=1$. After induction at $37^{\circ} \mathrm{C}$, induced cultures were washed twice with $1 \mathrm{~mL}$ of filter-sterilized PBS. Antibodies and no antibodies were added to these two strains as controls. Samples were analyzed using BD flow cytometry. The data shown on the left represent the geometric MFI. The data shown on the right represent the fluorescence intensity distribution of the bacteria analyzed in the experiment shown on the left. The mean of three independent assays is shown and error bars represent the standard deviation. ${ }^{\star \star \star \star} P<0.0001$ (Student's $t$ test).

A

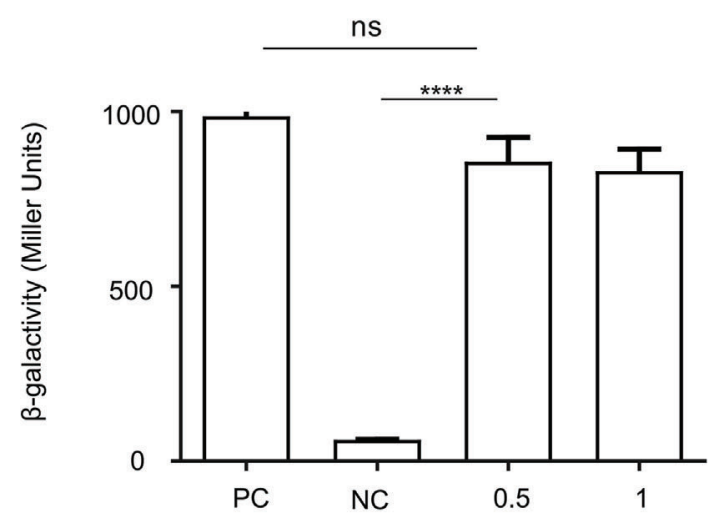

B

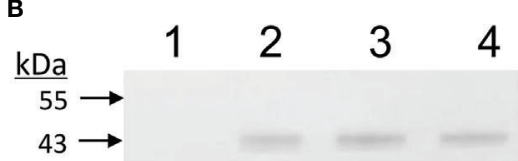

Anti-His

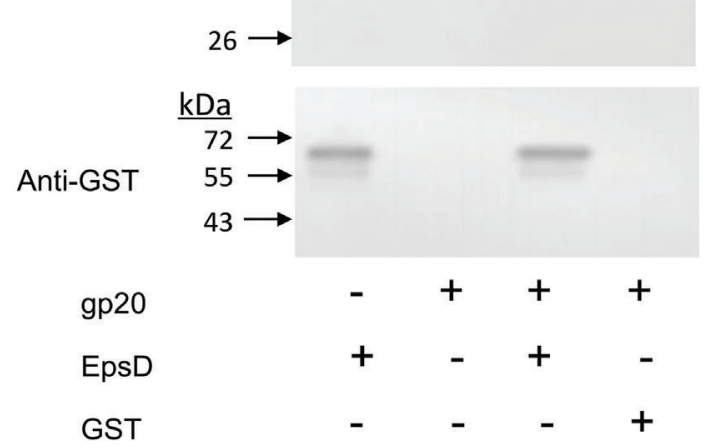

FIGURE 4 | Binding capacity of the VP2 tail filament protein with wild-type N16961 and N- $\Delta$ epsD, and analysis of the interactions of EpsD with gp20. (A) Analysis the gp20-EpsD interaction by BACTH. PC, positive control (leucine zipper of GCN4); NC, negative control (vector plasmid only). The resulting recombinant plasmid pair pT25-gp20/pT18C-EpsD was co-transformed into BTH101 cells, and the $\beta$-galactosidase activity was measured. ${ }^{* \star \star \star} P<0.0001$. ns, no significance. (Student's $t$ test). (B) Analysis of the interactions of EpsD with gp20 in vitro. Western blot analysis of His pull-down experiments was performed with GST-gp20 immobilized on $\mathrm{Ni}^{2+}$ resin, and the gp20 protein was incubated with GST for pull-down analysis as a negative control 0.3 mg of His6-tagged gp20 protein and 0.1 mg of GSTtagged EpsD protein were mixed, then the mixtures were rotated at room temperature for $2 \mathrm{~h}$, bound to $\mathrm{Ni} 2+$ resin and incubated for $1 \mathrm{~h}$ at $4^{\circ} \mathrm{C}$. Lane 1 : His-gp20; 2: GST-EpsD; 3: Pull-down of His-gp20 and GST-EpsD; 4 Pull-down of His-gp20 + GST. 
EpsM of T2SS plays a role in successful VP2 infection but does not affect VP2 binding to the host, suggesting that the intact T2SS structure probably acts as the phage DNA injection channel for crossing the outer and inner membranes of $V$. cholerae. The function of T2SS EpsD as a phage receptor has not been reported previously. Therefore, based on our study, the role of the T2SS in $V$. cholerae is expanded to the infection channel of the lytic phage, in addition to the transport of free proteins and release of filamentous phages.

\section{DATA AVAILABILITY STATEMENT}

The original contributions presented in the study are included in the article/supplementary material. Further inquiries can be directed to the corresponding author.

\section{REFERENCES}

Abendroth, J., Rice, A. E., McLuskey, K., Bagdasarian, M., and Hol, W. G. (2004). The crystal structure of the periplasmic domain of the type II secretion system protein EpsM from Vibrio cholerae: the simplest version of the ferredoxin fold. J. Mol. Biol. 338 (3), 585-596. doi: 10.1016/ j.jmb.2004.01.064

Bertozzi Silva, J., Storms, Z., and Sauvageau, D. (2016). Host receptors for bacteriophage adsorption. FEMS Microbiol. Lett. 363 (4), fnw002. doi: 10.1093/femsle/fnw002

Chiang, S. L., and Mekalanos, J. J. (2000). Construction of a Vibrio cholerae vaccine candidate using transposon delivery and FLP recombinase-mediated excision. Infect. Immun. 68 (11), 6391-6397. doi: 10.1128/iai.68.11.6391-6397.2000

Cianciotto, N. P., and White, R. C. (2017). Expanding Role of Type II Secretion in Bacterial Pathogenesis and Beyond. Infect. Immun. 85 (5), e00014-17. doi: 10.1128/IAI.00014-17

Davis, B. M., Lawson, E. H., Sandkvist, M., Ali, A., Sozhamannan, S., and Waldor, M. K. (2000). Convergence of the secretory pathways for cholera toxin and the filamentous phage, CTXphi. Science 288 (5464), 333-335. doi: 10.1126/ science.288.5464.333

Evans, F. F., Egan, S., and Kjelleberg, S. (2008). Ecology of type II secretion in marine gammaproteobacteria. Environ. Microbiol. 10 (5), 1101-1107. doi: 10.1111/j.1462-2920.2007.01545.x

Fan, F. X., Li, X., Pang, B., Zhang, C., Li, Z., Zhang, L. J., et al. (2018). The outermembrane protein TolC of Vibrio cholerae serves as a second cell-surface receptor for the VP3 phage. J. Biol. Chem. 293 (11), 4000-4013. doi: 10.1074/ jbc.M117.805689

Faruque, S. M., Albert, M. J., and Mekalanos, J. J. (1998). Epidemiology, genetics, and ecology of toxigenic Vibrio cholerae. Microbiol. Mol. Biol. Rev. 62 (4), 1301-1314. doi: 10.1128/MMBR.62.4.1301-1314.1998

Faruque, S. M., Islam, M. J., Ahmad, Q. S., Faruque, A. S., Sack, D. A., Nair, G. B., et al. (2005). Self-limiting nature of seasonal cholera epidemics: Role of hostmediated amplification of phage. Proc. Natl. Acad. Sci. U. S. A. 102 (17), 61196124. doi: 10.1073 /pnas. 0502069102

Frost, J. A., Kramer, J. M., and Gillanders, S. A. (1999). Phage typing of Campylobacter jejuni and Campylobacter coli and its use as an adjunct to serotyping. Epidemiol. Infect. 123 (1), 47-55. doi: 10.1017/s095026889900254x

Genin, S., and Boucher, C. A. (1994). A superfamily of proteins involved in different secretion pathways in gram-negative bacteria: modular structure and specificity of the N-terminal domain. Mol. Gen. Genet. 243 (1), 112-118. doi: $10.1007 / \mathrm{BF} 00283883$

Harvey, H., Bondy-Denomy, J., Marquis, H., Sztanko, K. M., Davidson, A. R., and Burrows, L. L. (2018). Pseudomonas aeruginosa defends against phages through type IV pilus glycosylation. Nat. Microbiol. 3 (1), 47-52. doi: 10.1038/s41564-017-0061-y

\section{AUTHOR CONTRIBUTIONS}

BK, HS and ML designed the study and wrote the paper. FF, ZheL and YF purified protein and performed the experiments. $\mathrm{JZ}$, ZheL, and JX provided technical assistance and contributed to the preparation of the figures. JL cryopreserved and saved the strains. YH contributed to diagram modification and protein purification in the process of article modification. All authors contributed to the article and approved the submitted version.

\section{FUNDING}

This work was supported by the National Key Basic Research Program (2015CB554201) from Ministry of Science and Technology of the People's Republic of China.

Hatfull, G. F., and Hendrix, R. W. (2011). Bacteriophages and their genomes. Curr. Opin. Virol. 1 (4), 298-303. doi: 10.1016/j.coviro.2011.06.009

Howard, S. P., Estrozi, L. F., Bertrand, Q., Contreras-Martel, C., Strozen, T., Job, V., et al. (2019). Structure and assembly of pilotin-dependent and independent secretins of the type II secretion system. PLoS Pathog. 15 (5), e1007731. doi: 10.1371/journal.ppat.1007731

Jensen, M. A., Faruque, S. M., Mekalanos, J. J., and Levin, B. R. (2006). Modeling the role of bacteriophage in the control of cholera outbreaks. Proc. Natl. Acad. Sci. U. S. A. 103 (12), 4652-4657. doi: 10.1073/pnas.0600166103

Johnson, T. L., Abendroth, J., Hol, W. G., and Sandkvist, M. (2006). Type II secretion: from structure to function. FEMS Microbiol. Lett. 255 (2), 175-186. doi: 10.1111/j.1574-6968.2006.00102.x

Judson, N., and Mekalanos, J. J. (2000). TnAraOut, a transposon-based approach to identify and characterize essential bacterial genes. Nat. Biotechnol. 18 (7), 740-745. doi: 10.1038/77305

Karimova, G., Pidoux, J., Ullmann, A., and Ladant, D. (1998). A bacterial twohybrid system based on a reconstituted signal transduction pathway. Proc. Natl. Acad. Sci. U. S. A. 95 (10), 5752-5756. doi: 10.1073/pnas.95.10.5752

Kazmierczak, Z., Gorski, A., and Dabrowska, K. (2014). Facing antibiotic resistance: Staphylococcus aureus phages as a medical tool. Viruses 6 (7), 2551-2570. doi: 10.3390/v6072551

Khan, S. R., Gaines, J., Roop, R. M., and Farrand, S. K. (2008). Broad-hostrange expression vectors with tightly regulated promoters and their use to examine the influence of TraR and TraM expression on Ti plasmid quorum sensing. Appl. Environ. Microbiol. 74 (16), 5053-5062. doi: 10.1128/ Aem.01098-08

Korotkov, K. V., and Sandkvist, M. (2019). Architecture, Function, and Substrates of the Type II Secretion System. EcoSal. Plus. 8 (2). doi: 10.1128/ ecosalplus.ESP-0034-2018

Korotkov, K. V., Sandkvist, M., and Hol, W. G. (2012). The type II secretion system: biogenesis, molecular architecture and mechanism. Nat. Rev. Microbiol. 10 (5), 336-351. doi: 10.1038/nrmicro2762

Ladant, D. (1988). Interaction of Bordetella pertussis adenylate cyclase with calmodulin. Identification of two separated calmodulin-binding domains. J. Biol. Chem. 263 (6), 2612-2618.

Liu, Z., Wang, H., Zhou, Z., Naseer, N., Xiang, F., Kan, B., et al. (2016). Differential Thiol-Based Switches Jump-Start Vibrio cholerae Pathogenesis. Cell Rep. 14 (2), 347-354. doi: 10.1016/j.celrep.2015.12.038

McCutcheon, J. G., Peters, D. L., and Dennis, J. J. (2018). Identification and Characterization of Type IV Pili as the Cellular Receptor of Broad Host Range Stenotrophomonas maltophilia Bacteriophages DLP1 and DLP2. Viruses 10 (6). doi: $10.3390 / \mathrm{v} 10060338$

Metcalf, W. W., Jiang, W., Daniels, L. L., Kim, S. K., Haldimann, A., and Wanner, B. L. (1996). Conditionally replicative and conjugative plasmids carrying lacZ alpha for cloning, mutagenesis, and allele replacement in bacteria. Plasmid 35 (1), 1-13. doi: 10.1006/plas.1996.0001 
Sun et al.

T2SS Mediates Phage Infection

Rakhuba, D. V., Kolomiets, E. I., Dey, E. S., and Novik, G. I. (2010). Bacteriophage receptors, mechanisms of phage adsorption and penetration into host cell. Pol. J. Microbial. 59 (3), 145-155. do: 10.33073/pjm-2010-023

Reidl, J., and Klose, K. E. (2002). Vibrio cholera and cholera: out of the water and into the host. FEMS Microbial. Rev. 26 (2), 125-139. doa: 10.1111/j.15746976.2002.tb00605.x

Russel, M. (1998). Macromolecular assembly and secretion across the bacterial cell envelope: type II protein secretion systems. J. Mol. Biol. 279 (3), 485-499. doit: 10.1006/jmbi.1998.1791 Sack, D. A., Sack, R. B., Nair, G. B., and Siddique, A. K. (2004). Cholera. Lancet 363 (9404), 223-233. do: 10.1016/s0140-6736(03)15328-7

Salmond, G. P., and Fineran, P. C. (2015). A century of the phage: past, present and future. Nat. Rev. Microbial. 13 (12), 777-786. doa: 10.1038/nrmicro3564

Sandkvist, M. (2001). Type II secretion and pathogenesis. Infect. Immune. 69 (6), 3523-3535. do: 10.1128/IAI.69.6.3523-3535.2001

Sikora, A. E., Zielke, R. A., Lawrence, D. A., Andrews, P. C., and Sandkvist, M. (2011). Proteomic analysis of the Vibrio cholera type II secretome reveals new proteins, including three related serine proteases. J. Biol. Chem. 286 (19), 16555-16566. doa: 10.1074/jbc.M110.211078

Simon, R., Priefer, U., and Pühler, A. (1983). A broad host range mobilization system for in vive genetic engineering: transposon mutagenesis in gramnegative bacteria. Nat. Biotechnol. 31 (5), 784-791. dor: 10.1038/nbt1183-784

Weil, A. A., and Ryan, E. T. (2018). Cholera: recent updates. Curt. Oping. Infect. Dis. 31 (5), 455-461. dor: 10.1097/QCO.0000000000000474
Yang, M., Lu, Z., Hughes, C., Stern, A. M., Wang, H., Zhong, Z., et al. (2013). Bile saltinduced intermolecular disulfide bond formation activates Vibrio cholera virulence. Proc. Natl. Acad. Sci. U. S. A. 110 (6), 2348-2353. dor: 10.1073/pnas.1218039110

Yang, Y., Shin, W., Zhong, Q., Chen, Q., He, X., Baker, J. L., et al. (2020). Development of a Bacteriophage Cocktail to Constrain the Emergence of Phage-Resistant Pseudomonas aeruginosa. Front. Microbial. 11, 327. dor: $10.3389 /$ fmicb.2020.00327

Chang, J., Li, W., Zhang, Q., Wang, H., Xu, X., Dian, B., et al. (2009). The core oligosaccharide and thioredoxin of Vibrio cholera are necessary for binding and propagation of its typing phage VP3. J. Bacteriol. 191 (8), 2622-2629. dor: 10.1128/JB.01370-08

Conflict of Interest: The authors declare that the research was conducted in the absence of any commercial or financial relationships that could be construed as a potential conflict of interest.

Copyright (C) 2021 Sun, Diu, Fan, Li, Fan, Chang, Huang, Li, Li, Xu and Kan. This is an open-access article distributed under the terms of the Creative Commons Attribution License (CC BY). The use, distribution or reproduction in other forums is permitted, provided the original authors) and the copyright owners) are credited and that the original publication in this journal is cited, in accordance with accepted academic practice. No use, distribution or reproduction is permitted which does not comply with these terms.

Frontiers in Cellular and Infection Microbiology | www.frontiersin.org

9

April 2021 | Volume 11 | Article 662344 